varying from 0.99 to 0.89 were prepared from simian virus (SV40) and phage PM2 DNAs. This $10 \%$ decrease in topological winding density results in a decrease of approximately $5 \%$ in the alkaline sedimentation coefficient, which can be understood in terms of a small negative change in the superhelix density of the denatured molecule. The data connect smoothly with similar results obtained by Sebring, Kelly, Thoren and Salzman (J. Virol., 8, 478; 1971) for DNAs with $A$ values from 0.6 to 0.15 . The range is completed by the inclusion of a point for $A \simeq 0$ computed from a relation proposed by Wang (Biopolymers, 9, 489; 1970). As $A$ decreases from 1 to 0 , the value of relative $s$ changes from 1 to 0.45 .

The $A$ values quoted here were determined on the assumption that for all naturally occurring closed circular DNAs, $A \leqslant 1$. This arises from the interpretation of the experimental results obtained from studies on the binding of intercalating dyes to superhelical DNA in accordance with the proposal by Lerman ( $J$. cell. comp. Physiol., 64, suppl. 1, 1; 1964) and Fuller and Waring (Ber. Bunsenges. phys. Chem., 86, 805; 1964) that intercalating dyes unwind the DNA helix. The observed data are then consistent with a model in which the superhelix density $\sigma$ is initially less than 0 and $A$ is not greater than 1 .

The recent assertion by Paoletti and LePecq (J. molec. Biol., 59, 43; 1971) that ethidium bromide winds up the DNA helix has the corollary that $A \geqslant 1$ in naturally occurring closed circular DNAs. Schmir et al. are able to refute this suggestion completely. First, they point out that the data of Sebring et al. were derived from replicating intermediates of SV40 DNA which were extensively unwound while nicked during the replicating process. There can therefore be no doubt that for these DNAs, $A \ll 1$. In addition, the point computed by Wang is unaffected by considerations of winding or unwinding the helix. Second, they have carried out dye titrations with a closed circular replicative mitochondrial DNA before and after removal of a short progeny strand (Révet, Schmir and Vinograd, Nature new biol., 229, 10; 1971). These experiments indicated quite clearly that less ethidium bromide was required to relax the supercoiled DNA molecule in the presence of the progeny strand than was required after its removal. The removal was achieved in such a way that the parent DNA did not become denatured and was able to rewind into its original structure. The results of Schmir et al. indicate unequivocally that the insertion of a displacing progeny strand and the binding of ethidium bromide act in the same direction, that is, they both unwind the DNA helix.
Schmir et al. conclude therefore that the initial superhelix density must be negative, $A$ is less than 1 and the sense of the supercoils in natural closed circular DNA is negative, not positive as proposed by Paoletti and LePecq.

The results obtained during the past ten years on the binding of certain dyes to superhelical DNAs can be interpreted almost without exception in terms of a model in which the bound dye produces an unwinding in the DNA double helix. In view of the extreme sensitivity of superhelical DNA to small changes in its structure, this technique might in the future provide a means of distinguishing between the different modes of interaction of DNA and a variety of dye molecules.

\section{Torpor in an Andean hummingbird}

from our Animal Ecology Correspondent THE attraction of hummingbirds to physiologically-inclined ecologists is easy to understand. How do these tiny, active creatures manage to survive in the inhospitable habitats of some of the world's highest mountains? Calder and Booser (Science, 180, 751; 1973) found that nocturnal torpor during incubation was not uncommon at times of energy depletion. When the ambient temperature was low and heavy rain precluded nocturnal foraging, a gradual lowering of the body temperature ensured the sufficiency of the bird's energy reserves to see it through the night.

Another physiological adaptation of obvious survival value has been reported by Carpenter (Science, 183, 545; 1974) who worked with a population of the hillstar hummingbird Oreotrochilus estella, which lives at a height of between $3,800 \mathrm{~m}$ and $4,300 \mathrm{~m}$ in the high Andes. By day the birds forage for nectar from a variety of local and exotic plants. At night they roost communally in caves and can readily be observed with little disturbance. Carpenter recorded body temperatures from a small group of birds: most were recorded between one and eight times during either the summer or winter study periods; a few were recorded in both summer and winter. Torpor was defined as a significant drop from the normal body temperature of about $30^{\circ} \mathrm{C}$. In summer the average duration of nocturnal torpor was $7 \mathrm{~h}$ and during the winter $10 \mathrm{~h}$. This difference is significant in spite of the fact that winter nights were 15 to 90 min longer than summer nights. Calder and Booser reported that body temperature during nesting torpor never sank below $6.5^{\circ} \mathrm{C}$. The same was true in Carpenter's study, even though ambient temperature sometimes dropped to $3^{\circ} \mathrm{C}$.

Why is torpor used more extensively

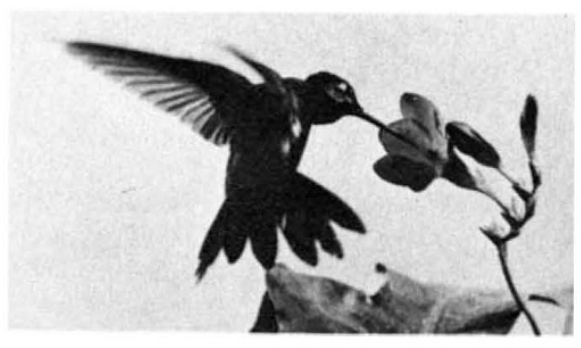

Zoological Society of London Stripe breasted starthroated hummingbird (Heliomaster squamosus), a different species of hummingbird from that described by Carpenter but it shows the characteristic feeding behaviour of these birds.

by the birds in winter? One of the winter groups of birds lives in an area surrounded by blooming Eucalyptus trees, from which they fed heavily. Incidence and duration of torpor was the same as in other local populations with less well endowed larders. It seems as if energy depletion is not a cue for torpor. Nor does temperature seem to be the trigger. In summer the ambient temperatures in the roosts varied from $3.5^{\circ} \mathrm{C}$ to $13^{\circ} \mathrm{C}$, and in winter from $3.0^{\circ} \mathrm{C}$ to $13 \mathrm{C}^{\circ}$. This high winter temperature is thought to be exceptional; winter roost temperatures are normally near freezing.

Carpenter suggests that although the difference between summer and winter ray length is brief, it is sufficient to act as a photoperiodic cue for a circannian rhythm. Such a rhythm would have survival value for a species living in an area where nocturnal temperature is seasonally low and in which the day time temperature gives no indication of the ensuing night time level. By entering torpor as soon as it roosts, a bird is capable of regulating its body temperature for long periods should the ambient temperature drop much below $6.5^{\circ} \mathrm{C}$-a clever way of extending fuel contingency reserves without increasing fuel capacity.

\section{Probing the secrets of ionic channels}

from a Correspondent

SINCE Galvani's fortuitous joining of brass hook, iron plate and frog in 1786, the lure and mystery of 'animal electricity' have been reduced to the molecular secrets of so-called cation channels, which intermittently allow these ions to cross membranes. Recent chemical and electrical probes of the structure of these channels were the main topics of a meeting at the Royal Society on March 13-14.

The simplest model of a channel is a pore, a few Ångstroms in diameter, capped by one or more gates which open or close the pore depending on the recent history of the voltage across 